\title{
Littérature féminine et littérature pour filles au 19e siècle: Mobiles
}

\author{
Bénédicte Monicat
}

The little girl becomes a reader in the 19th century. Numerous women writers wish to prepare the child for her future as a woman by writing novels dominated by educational paradigms. The little girl, now a heroine, is often held in rigid frames of conventional femininity, though in works of fiction she also becomes the symbol of change. From the most rigidly codified texts, to the most inspired works, the stories written by women writers explain to girl readers the complexity and transformations of women's history.

L'enfance des filles est au $19^{\mathrm{e}}$ siècle un objet de réflexion dont les enjeux critiques sont liés aux grandes mutations de l'époque et touchent à des questions institutionnelles (l'école), économiques (le travail des femmes), sociales (la famille) ${ }^{i}$. La petite fille devient alors l'interlocutrice privilégiée de bien des écrivains particulièrement soucieux de cultiver en elle ce que l'on pourrait nommer « l'êtrefemme-en-fille », à savoir le devenir de femme de l'enfant. Il faut en effet se défier en elle de ce que Pierre Péju appelle « l'être-petite-fille », cette « façon de s'esquiver des rôles (féminins) et du sérieux, mais aussi des genres, des sexes » (126). Puisque les femmes ont à charge la petite enfance et l'éducation des filles, nombreuses sont celles qui pratiquent alors la fiction comme outil éducatif, sinon littéraire. Aborder la question de la littérature pour filles au $19^{\mathrm{e}}$ siècle, c'est donc aussi aborder celle de la littérature féminine, d'une littérature qui nous est encore peu familière. Partie prenante d'une nouvelle mobilité socio-économique dont témoignent tout au long du siècle les contributions importantes des femmes aux institutions éducatives (que ce soit dans leurs capacités de théoriciennes, d'administratrices, ou d'institutrices), l'on peut se demander si ce domaine de la créativité littéraire féminine ne cultive pas aussi une mobilité culturelle dont les retombées permettraient, entre autres, de repenser l'expérience de la « séparation des genres » et ses transformations.

Répondre à une telle interrogation n'est pas une tâche aisée tant d'un point de vue quantitatif (le corpus en question est extrêmement vaste) que qualitatif: comment rendre compte d'un mode d'écriture qui multiplie ses incarnations mais qui répond à des raisons d'être pour la plupart très sommairement stipulées (il faut former les filles aux rôles et aux fonctions qui seront les leurs) et banalement formulées (il s'agit d'éduquer ou d'instruire les filles en les amusant)? Pour mieux saisir tant la fixité que le dynamisme de tels écrits, pour en évaluer les effets, je m'appuierai sur les trois acceptions majeures d'un terme qui permet d'appréhender plus aisément la complexité du phénomène: le mobile. Sa première définition ${ }^{\text {ii }}$ 
réfère à « tout corps qui se meut ou est mû, considéré dans son mouvement 》. Il est également " ce qui fournit une impulsion, un mouvement », et enfin un « ensemble d'éléments construits en matériaux légers et agencés de telle sorte qu'ils prennent des dispositions variées sous l'influence du vent ou de tout autre moteur $»$.

La stabilité du foyer et une existence casanière étant présentées à la petite fille comme des idéaux de vie, on pourrait aisément supposer que les jeunes héroïnes des ouvrages que leur consacrent les écrivaines sont le plus souvent représentées et immobilisées dans un cadre familial et familier. Ce n'est pas le cas. Comme je l'ai noté ailleursiii, elles y font très souvent l'expérience de voyages tant domestiques que lointains. Si l'aventure leur échoie de manière passive (les garçons peuvent choisir de partir, les filles suivent et subissent pour la plupart le déplacement: elles sont mues avant que de se mouvoir), elle ne fait pas moins partie du paysage littéraire réservé aux filles et aux jeunes filles: robinsonnades, voyages exotiques, départs en vacances et autres escapades estivales en rythment la production. Ce corps est-il cependant " considéré dans son mouvement "? Le mouvement n'est-il pas plutôt pour la jeune héroïne le fait d'un déplacement qui, ne pouvant se permettre d'être « déplacé », ne dérange pas le monde tel qu'il doit être ordonné pour les filles et les femmes? Paradoxe apparent, la mobilité du corps de l'héroïne intensifie souvent l'immobilité de son être: plus le monde s'ouvre à elle, plus son personnage doit être défini dans la permanence d'une identité féminine fixe. Au réalisme des lieux évoqués fait donc pendant l'artifice de personnages dont l'intégrité idéologique doit être préservée d'une représentation authentique de leur présence au monde.

Il peut de fait alors être plus risqué de faire se mouvoir l'héroïne dans un imaginaire fantaisiste, puisque ce dernier permet des expériences, des explorations et des questionnements que n'autorise pas le texte " réaliste ». Dans un ouvrage intitulé Esquisses morales, contes aux jeunes personnes, Laure Bernard met ainsi en scène une petite Léonie de dix ans que l'imagination emporte à travers les siècles au fil de lectures où, d'Andromaque à Ulysse, l'enfant assume l'identité des sujets de l'histoire lue. Son institutrice la met en garde contre les dangers qui pourraient en résulter: "Tâchez de ne pas tomber dans le monde comme dans un pays étranger, dont vous ne connaîtriez ni les usages ni la langue » (109). Pour Bernard comme pour tant d'autres, telle Laure Surville évoquée ci-dessous, il est urgent de maintenir les possibilités d'échappées de l'imaginaire dans les contours d'une écriture didactique. Pour celles qui, au contraire, cultivent avec bonheur de tels transports, comme George Sand mentionnée plus loin, il s'agit de jouer du plaisir de la traduction, de compliquer les parcours du sens, et de fonder l'intelligence du texte sur une poétique qui libère la lecture des savoirs culturels ou linguistiques obligés. D'un côté donc, une volonté de définir distinctement le réel et la fiction pour ne pas déstabiliser la fonction première du récit et l'ancrer dans un mode d'écriture statique supposé contrôler les errances de la lecture, refusant de cultiver les vagabondages des jeunes lectrices dans un imaginaire pouvant si vite devenir suspect. De l'autre, une mobilité qui tient à l'esthétique de textes où cohabitent, se mêlent et se brouillent divers registres (merveilleux, scientifique, moralisateur, philosophique, etc.). 
C'est ainsi qu'avec les Contes d'une grand'mère de George Sand, nous parcourons les univers du monde physique, artistique, spirituel (de la botanique à la musique, de la géologie au bouddhisme) au fil d'expériences formatrices et transformatrices fondées dans une éthique et une esthétique du mouvement. Sans pour autant éliminer des principes communs telle la notion du « devoir » (devoir de travailler par exemple), chacun y est encouragé à explorer, et la curiosité et la créativité y sont encouragées: « Rêvez, imaginez, faites du merveilleux, vous ne risquez pas d'aller trop loin, car l'avenir du monde idéel auquel nous devons croire dépassera encore de beaucoup les aspirations de nos âmes timides et incomplètes » (« Le chien et la fleur sacrée »103). Le transport dans un imaginaire valorisé fait fi des ancrages idéologiques de ce que peut signifier « une vie de fille » ainsi qu'en témoigne l'une des héroïnes de Sand, Diane la jeune artiste peintre du « Château de Pictordu », qui s'interroge: « Elle savait bien qu'au-delà du métier qui assurait son indépendance et sa dignité, il y avait un grand essor à prendre: mais pourrait-elle jamais entrer dans les conditions de ce développement? Pourrait-elle voyager, connaître, sentir? Secouer l'entourage, l'habitude, le devoir de chaque jour " (107)? Guidée par la figure inspiratrice de la mère morte, l'héroïne deviendra artiste. De nouveaux modèles et modes de vie s'offrent à la petite fille.

La plupart des écrivaines ne veulent cependant ni encourager ni assumer l'envol artistique par le biais de la fiction. Elles tentent plutôt d'en contrôler les effets potentiellement pernicieux. Ce que Christine Planté note dans La Petite soeur de Balzac au sujet de Laure Surville peut leur être plus largement appliqué: «Si elle incarne l'écrasement de la femme par les conventions sociales, c'est de façon infiniment moins tragique que la petite sœur de Shakespeare dans la fable imaginée par Woolf, mais à la manière du XIXe siècle bourgeois: dans le triomphe apparemment serein d'une certaine médiocrité » (154). Surville publie en 1854 La Fée des nuages ou La Reine Mab, ouvrage qui s'ouvre sur un avant-propos où l'auteure oppose fiction (le conte de fées, l'imagination) et réalité (l'instruction, la vérité) en minimisant la portée de la première: « La jeunesse ne croit pas aux fées et ne prend pas des rêves pour des vérités ». L'héroïne du livre est une jeune Éliane pour qui les transports cérébraux sont la « vraie vie »: « Les objets qu'Éliane a sans cesse sous les yeux, les sites grandioses qui l'entourent, les conversations qu'elle entend, les récits merveilleux qu'on lui fait, l'absence de toute amie de son âge; tout concourut à la jeter avant le temps dans le monde des idées, monde enchanté, sans limites ni entraves, où la petite a des ailes et se crée déjà des fêtes » (30-31). Sous l'égide de la shakespearienne fée Mab, Éliane parcourt chaque nuit les univers entrevus le jour sur les bancs de l'école du couvent. Ces expériences nocturnes présentent souvent l'enfant comme une spectatrice plus qu'une exploratrice, mais Éliane vit au plus près les leçons à impartir par le biais des stratagèmes de la fiction, au plus près et finalement de trop près. Miniaturisée pour une « promenade sous le gazon » qui lui fait découvrir la vie des insectes, transportée sur une montagne d'où elle peut voir jusqu'à l'océan et dialogue avec une goutte d'eau ondine qui lui fait de la « théorie des eaux du globe » (94) un récit autobiographique, Éliane finit ses voyages entraînée par la fée alors ivre dans une « danse des astres » (223) qui laisse la petite fille 
elle-même incapable de contrôler le tourbillon du savoir qui brûle en elle. L'héroïne retourne alors au château familial où le docteur pronostique: « Sa fée ne la reprendra pas au milieu de sa famille, et les romans, que votre fille fera maintenant seront tous tirés de son cœur et non de son esprit » (245). Un « vrai » voyage achèvera de guérir la convalescente, voyage où le « réel » sera jugé supérieur aux visions de l'esprit: « Là, encore, la réalité tuait la fiction » (247). Et l'on pourrait sans doute en dire de même des écrits de tant de ces femmes qui, à l'instar de Laure Surville, et par contraste avec George Sand, ne peuvent pas - ou ne veulent pas - se laisser prendre aux pièges d'une écriture dont les élans seraient par trop contagieux. Il faut se protéger de l'《 être-fille-en- femme », concept foncièrement perturbateur, sinon subversif.

De manière intéressante, le récit fantaisiste rejoint alors le récit ancré dans la représentation d'un réalisme conjugué au quotidien. Il s'agit dans le premier cas de maintenir la petite fille dans un univers imaginaire qui ne s'écarte pas des devoirs familiers et dans le second de faire du lieu des devoirs familiers un espace évocateur où la femme et sa fille ne sont pas figées dans l'absolu des formules. On pense par exemple à ces personnages de mères qui, dans les ouvrages de la comtesse de Ségur, courent et rient avec leurs enfants dans des parties de cachecache où les moins jeunes ne sont pas les moins emportées par le jeu. On pense à ces mouvements de tous les jours que sont les jeux de la petite fille, au défoulement physique qui les accompagne parfois ${ }^{i v}$. Si les récits sont souvent entravés, les corps n'y sont pas nécessairement immobilisés. On pense enfin à l'expérience quotidienne de la nature qui peut bien sûr se résumer en une routine banale et anodine (visites au jardin du Luxembourg, moments de récréation) mais qui a également le potentiel de révéler certaines dimensions capitales de ce qui définit la petite fille: son désir de mobilité. Les héroïnes de Zénaïde Fleuriot, comme l'a noté Amy Falk, ont ainsi une relation privilégiée à des lieux (la forêt, l'océan) qui évoquent une liberté ailleurs réduite, qui sont des promesses d'horizons élargis. Dans ces lieux peut se produire le glissement vers d'autres territoires, tels ceux dont fait état l'héroïne éponyme de Thérèse Bentzon, Geneviève Delmas, lorsqu'elle se retrouve dans une nature où l'enfant se transforme en conteuse: « Aucun obstacle ne m'arrêtait; je créais autour de moi le fantastique, et je réussissais à le faire voir aux autres. C'était, en d'autres termes, mon domaine de prédilection » (43). La liberté vécue corporellement se traduit donc souvent pour la petite fille en une activité qui nourrit la créativité. Et c'est de fait parce que la femme dans son enfance est souvent dite « mobile » de nature que l'on s'attache avec tant d'effort à la fixer dans des manières d'être prévisibles et contrôlées. $C$ 'est ici que la deuxième acception du terme « mobile » prend toute son importance.

« Ce qui fournit une impulsion, un mouvement ». Une telle définition est à considérer sous deux aspects: d'une part, les justificatifs qui décident de la direction donnée au récit, d'autre part, ce qui fait avancer l'histoire. Le premier aspect permet de mieux saisir le cadre dans lequel l'écriture des femmes se fait acte légitime dans son rapport avec les jeunes lectrices, ses destinataires explicites. Le second aspect permet d'examiner en quoi la marche du récit conforte certes, mais 
fragilise aussi les données de sa raison d'être, ce à la fois pour les lectrices et les auteures. Dans un cas comme dans l'autre, qu'il s'agisse de la volonté justificatrice de l'écrit ou de son élaboration en récit, les " modèles » souffrent de ce qui leur échappe nécessairement. Les écrivaines qui, les plus nombreuses, rejettent explicitement les visées littéraires d'une écriture alors forte (et lourde) de son didactisme et de ses diverses fonctions formatrices formulent certes leur but clairement, mais il s'avère que « raconter une histoire » est toujours un projet à risque. Le mobile formateur, souvent instructif et toujours moralisateur, se nourrit en effet de ce qui le menace: l'imperfection, la résistance aux modèles prônés, le danger de certains comportements, et surtout une « nature » qui défie la " culture »du féminin de convention et qui est donc à corriger. La petite fille " caméléon » que critique Mme Mallès de Beaulieu dans un conte intitulé "La petite fille sans caractère » ne trouve ainsi aucune objection et aucun déplaisir à se comporter en « petit garçon manqué 》 et à jouer avec les paysans qui sont ses compagnons: « Elle grimpait sur les arbres comme un écureuil, défiait à la course le petit pâtre le plus agile, n'avait peur de rien, avait enfin les manières et la rudesse des compagnons de ses plaisirs » (194). Elle apprendra bien sûr que ce n'est pas ainsi qu'une demoiselle se fait respecter et que les paysans ne sont pas ses égaux. La " nature » de l'enfant étant imparfaite, les " métamorphoses" et autres " conversions » prévalent dans les scénarios présentés aux jeunes lectrices, leurs seules alternatives étant pour les héroïnes mises en scène des punitions exemplaires (mort, défiguration, abandon parmi d'autres).

La mobilité même se fait alors mobile. L'inconstance, le désordre, la légèreté et la dissipation cèdent à la stabilité, la régularité, l'application et surtout, la soumission. La fille est envisagée, telle l'héroïne que Mathilde Bourdon met en scène dans La Fille du fermier, comme un « instrument mobile » (22) qui défie et menace des ordres hiérarchiques sociaux et sexués voulus statiques. Le schéma inverse est également commun mais participe du même projet. Il postule que dans un monde foncièrement instable, seule la permanence des qualités attribuées à la fille (femme en devenir) préserve du chaos toujours menaçant. Les récits peuvent alors user d'un rocambolesque faisant passer l'héroïne par des séries d'épreuves et de déracinements qui sont mis au service d'une morale et d'une " philosophie » posées dans une évidence et une permanence indiscutées (l'« être-femme-en-fille »). Sophie de Cantelou résume ce qui sous-tend alors souvent l'intrigue: la femme est dès son enfance « une véritable machine à douleur » (150). Tout devient alors possible, tout est imaginable: l'impulsion narrative se soumet à la volonté de démontrer que subir et accepter est la seule réponse à envisager dans le contexte de ces fictions. L'encadrement utilitariste et moraliste qui mobilise de tels récits aboutit en une mécanique narrative qui laisse en apparence peu de place à une créativité s'assumant en tant que telle. Elle est cependant aussi de l'ordre du jeu, parce que mécanique. Accumulation de péripéties, scénarios figés et formules attendues: le plaisir ou tout au moins le processus de la lecture n'est-il pas aussi pour la petite fille de parcourir ces chemins bornés où l'on sait ne pas devoir se perdre mais où l'on peut licitement considérer ce dont on est " protégée »? 
Corps mobiles réifiés dans des scénarios prétextes, mais également incarnations allégées par un factice familier, les figures imposées présentées aux lectrices finissent par se transformer en d'étranges objets que l'on sait échapper à l'emprise totalisante de ces projets d'écriture. Je fais ici allusion à la troisième définition du terme " mobile », cet " ensemble d'éléments construits en matériaux légers et agencés de telle sorte qu'ils prennent des dispositions variées sous l'influence du vent ou de tout autre moteur $»$. Si le processus de la lecture génère ces pulsions, ou impulsions, l'engagement dans l'écriture est le point critique où s'actualisent hors de la négation, dans la friction - dans la fiction - les sujets femmes pour, à travers, et par contraste avec les filles. Hors de la négation puisqu'une matière première partagée investit l'acte d'écrire de promesses littéraires, quel que soit effectivement son aboutissement. Ainsi, nombreuses sont celles qui, telle Mme Mallès de Beaulieu dans ses Contes d'une mère à sa fille (1817), disent travailler une matière commune et des idées sans originalité. L'auteure fera acte, nous dit-elle, dans la " manière de présenter ». L'art du récit, devenu l'enjeu du projet de l'écrivaine, définit ce dernier dans ses dimensions esthétiques. Hors de la négation également pour ce qui est de la " mobilité rhétorique » surtout caractéristique des ouvrages les plus ludiques de ce corpus (" Jamais une mère n'oserait dire tant de folies à ses enfants ", déclare Delphine de Girardin dans la préface à ses Contes d'une vieille fille à ses neveux), mais hors de la négation aussi jusque dans les textes aux combinatoires les plus codifiées, dans les ouvrages aux fins les plus claires comme elles le sont dans celui de Laure Surville.

Éliane s'y remet de ses maladies de l'imaginaire en revenant au sein de la famille, auprès d'une mère qui assure la prédominance du sentiment dans la vie de sa fille. Cette dernière est maintenant soutenue par une foi religieuse qui autorise et entraîne l'exaltation de la jeune héroïne comme Mab le faisait autrefois. Si la jeune fille finit par être « guérie de ses hallucinations » de petite fille (250), Éliane devenue femme « resta toujours exaltée » (250-51). Jusque dans ses vieux jours, elle cache des élans qu'elle sait inconvenants mais qu'elle ne veut ni ne peut étouffer: «S Son imagination volait au-devant d'inquiétudes imaginaires qui lui triplaient ses peines, mais aussi ses joies, de sorte que l'incorrigible femme n'eût pas changé les agitations perpétuelles de son âme pour le repos » (251). Banale histoire sans doute que celle d'une vie ainsi entravée, témoignage également commun qu'un récit refusant de se laisser emporter mais disant ses tentations. Il faut préserver l'écrivaine, l'héroïne et la lectrice des risques du départ, comme l'un des personnages de Joséphine Colomb se l'entend dire: « Tu as raison, ma Jeanne! Ne t'en va pas... Une fois qu'on est parti, vois-tu, on n'est jamais sûr de revenir " ("La jolie Jeanne » 113). Que les récits destinés à un jeune lectorat féminin fassent de la mobilité de l'être - et donc de « l'être-fille-en-femme »un anathème ou un bonheur selon les mobiles agencés par leurs auteures, ces dernières transforment les données de l'expérience féminine dans un rapport à l'écriture qui échappe aux simples permanences expliquant la présence de l'écrit. Une fois parties en écriture, une fois engagées dans la lecture, il n'y a pour les femmes et les filles plus de retour possible, seuls des cheminements hasardeux. 
Les transformations dont témoigne 1 'histoire des femmes au $19^{\mathrm{e}}$ siècle doivent être pensées jusque dans ces histoires-mobiles, objets et expériences clés d'une féminité qui est toujours à raconter.

\section{Notes}

i Voir, parmi d'autres, les ouvrages de Marie-Françoise Lévy, De mères en filles: l'éducation des françaises 1850-1880, et de Colette Cosnier, Le silence des filles: de l'aiguille à la plume. Pour une réflexion générale sur l'enfance, voir l'Histoire de l'enfance en Occident: du XVIIIe siècle à nos jours, sous la direction d'Egle Becchi et de Dominique Julia.

ii Ces définitions sont celles du dictionnaire Petit Robert.

iii Voir « Off on Adventures: Robinsonnades, Long Voyages, and Escapades for Little Girls ».

iv L'exercice physique est communément recommandé aux filles, l'un des défauts souvent décrié à leur égard étant la nonchalance, synonyme de délicatesse ou de paresse. Voir par exemple l'ouvrage pour les jeunes filles que Marguerite du Parquet consacre aux jeux (Jeux et exercices des jeunes filles, 1856) qui y sont considérés comme bénéfiques tant physiquement qu'intellectuellement. Ceci évidemment en tout bien tout honneur et avec mesure, comme le précise Mme Guizot: "En laissant à nos filles le mouvement nécessaire à leur âge, nous ne provoquons pas chez elles les essais de force et de hardiesse; malgré 1'heureuse innovation du pantalon qui a manqué à nos mères, nulle de nous n'exerce sa fille à la culbute » (131).

\section{Bibliographie}

Becchi, Egle et Dominique Julia. Histoire de l'enfance en Occident: du XVIIIe siècle à nos jours. Paris: Seuil, 1998.

Bentzon, Thérèse. Geneviève Delmas. Paris: Hetzel, s.d.

Bernard, Laure. Esquisses morales, contes aux jeunes personnes. Paris: Louis Janet, s.d.

Bourdon, Mathilde. La fille du fermier. Lille: Lefort, 1853.

Cantelou, Sophie de. Risette, histoire d'une petite fille. Rouen: Mégard, 1892.

Colomb, Joséphine. Histoires de tous les jours. 8e éd. Paris: Hachette, 1909.

Cosnier, Colette. Le silence des filles: de l'aiguille à la plume. Paris: Fayard, 2001.

Du Parquet, Marguerite. Jeux et exercices des jeunes filles. Paris: Hachette, 1856.

Falk, Amy Cartal. Zénaïde Fleuriot's Inspired Amazons: Reinventing the World in Nineteenth-Century Children's Literature (France). The Pennsylvania State University, 2002.

Girardin, Delphine de. Contes d'une vieille fille à ses neveux. Paris: Librairie Nouvelle, s.d. 
Guizot, Mme. Éducation domestique ou lettres de famille sur l'éducation. 6e éd. Paris: Didier, 1881.

Lévy, Marie-Françoise. De mères en filles: l'éducation des Françaises 18501880. Paris: Calmann-Lévy, 1984.

Monicat, Bénédicte. « Off on Adventures : Robinsonnades, Long Voyages, and Escapades for Little Girls ». Women Seeking Expression. France 1789-1914. Eds. Rosemary Lloyd and Brian Nelson. Monash Romance Studies 6 (2000): 68-85. Péju, Pierre. La petite fille dans la forêt des contes. Paris: Laffont, 1981.

Planté, Christine. La petite soeur de Balzac. Essai sur la femme auteur. Paris: Seuil, 1989.

Sand, George. Contes d'une grand'mère. Première série. Grenoble: Editions de l'Aurore, 1982.

Surville, Laure. La fée des nuages ou La reine Mab. Paris: Giraud, 1854. 